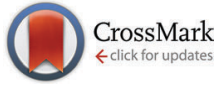

Cite this: J. Mater. Chem. B, 2015, 3, 6293

\title{
Visualisation of dual radiolabelled poly(lactide-co- glycolide) nanoparticle degradation in vivo using energy-discriminant SPECT
}

\author{
J. Llop,,$^{\star a}$ P. Jiang, ${ }^{b}$ M. Marradi, ${ }^{c}$ V. Gómez-Vallejo, ${ }^{d}$ M. Echeverría, ${ }^{c}$ S. Yu, ${ }^{b}$ \\ M. Puigivila, ${ }^{a}$ Z. Baz, ${ }^{a}$ B. Szczupak, ${ }^{e}$ C. Pérez-Campaña, ${ }^{a}$ Z. Mao, ${ }^{b}$ C. Gao*b and \\ S. E. Moya*c
}

\begin{abstract}
The determination of nanoparticle (NP) stability and degradation in vivo is essential for the accurate evaluation of NP biodistribution in medical applications and for understanding their toxicological effects. Such determination is particularly challenging because NPs are extremely difficult to detect and quantify once distributed in a biological system. Radiolabelling with positron or gamma emitters and subsequent imaging studies using positron emission tomography (PET) or single-photon emission computerised tomography (SPECT) are some of the few valid alternatives. However, NPs that degrade or radionuclides that detach or are released from the NPs can cause artefact. Here, submicron-sized poly(lactide-COglycolide) nanoparticles (PLGA-NPS) stabilised with bovine serum albumin (BSA) were dual radiolabelled using gamma emitters with different energy spectra incorporated into the core and coating. To label the core, ${ }^{111}$ In-doped iron oxide NPs were encapsulated inside PLGA-NPs during NP preparation, and the BSA coating was labelled by electrophilic substitution using ${ }^{125} \mathrm{I}$. After intravenous administration into rats, energy-discriminant SPECT resolved each radioisotope independently. Imaging revealed different fates for the core and coating, with a fraction of the two radionuclides co-localising in the liver and lungs for long periods of time after administration, suggesting that NPs are stable in these organs. Organ harvesting followed by gamma counting corroborated the SPECT results. The general methodology reported here represents an excellent alternative for visualising the degradation process of multi-labelled NPs in vivo and can be extended to a wide range of engineered NPs.
\end{abstract}

Received 15th June 2015, Accepted 25th June 2015

DOI: $10.1039 / \mathrm{c} 5 \mathrm{tb} 01157 \mathrm{~d}$

www.rsc.org/MaterialsB

\section{Introduction}

The pharmacokinetics (PK) and biological fate of nanoparticles (NPs) need to be known to accurately assess their toxicological effects $^{1}$ and therapeutic efficacy ${ }^{2}$ in biomedical applications. However, NPs are extremely difficult to detect and quantify once distributed in a biological system. In preclinical studies, organs are often harvested at pre-determined time points after

\footnotetext{
${ }^{a}$ Radiochemistry and Nuclear Imaging, CIC biomaGUNE, Paseo Miramón 182, 20009, San Sebastián, Guipúzcoa, Spain. E-mail: jllop@cicbiomagune.es; Tel: +34 943005333

${ }^{b}$ MOE Key Laboratory of Macromolecular Synthesis, Functionalization, Department of Polymer Science, Engineering, Zhejiang University, Hangzhou, 310027, China. E-mail: cygao@zju.edu.cn; Tel: +8657187951108 'Soft Mater Nanotechnology Laboratory, CIC biomaGUNE, Paseo Miramón 182, 20009, San Sebastián, Guipúzcoa, Spain. E-mail: smoya@cicbiomagune.es; Tel: +3494300 5311

${ }^{d}$ Radiochemistry Platform, CIC biomaGUNE, Paseo Miramón 182, 20009, San Sebastián, Guipúzcoa, Spain

${ }^{e}$ Nuclear Imaging Platform, CIC biomaGUNE, Paseo Miramón 182, 20009, San Sebastián, Guipúzcoa, Spain
}

administration or incorporation of the NPs and, depending on their nature, are then directly visualised by imaging techniques such as transmission electron microscopy (TEM) ${ }^{3}$ and confocal Raman microscopy ${ }^{4}$ or indirectly quantified by ion beam microscopy ${ }^{5}$ or inductively coupled plasma mass spectrometry (ICP-MS). ${ }^{6}$ These techniques require the sacrifice of large numbers of experimental animals to ensure statistical power, can only be applied to certain NPs, and not all of them are quantitative. New in situ methodologies are required for PK and fate studies in vivo.

NPs can be labelled with radionuclides (e.g., positron or gamma emitters) to reduce animal use and generate quantitative data in fate studies. Radiolabelled NPs are then commonly detected in vivo using ultra-high sensitivity imaging modalities such as positron emission tomography (PET) or single-photon emission computerised tomography (SPECT). ${ }^{7}$ A wide variety of procedures have been developed for the incorporation of positron or gamma emitters into NPs including direct ion ${ }^{8-10}$ or neutron activation, ${ }^{11-13}$ the attachment of bifunctional chelators (BFCs) followed by complexation with a radiometal, ${ }^{14-16}$ the 
incorporation of a pre-labelled tag into NPs, ${ }^{17-19}$ or the incorporation of a labelled precursor during NP synthesis. ${ }^{20}$ Although the spatiotemporal data gathered by radiolabelling and subsequent imaging are useful, the radiochemical integrity or chemical stability of the NPs is not reported: NPs that degrade or radionuclides that detach or are released from the NPs can cause artefact.

Ideally, in vivo radiochemical integrity and NP stability should be determined when investigating NP biodistribution. Moreover, knowledge of NP stability or degradation is fundamental when assessing the biological fate of NPs, since NP degradation can significantly alter toxicological endpoints. ${ }^{21}$ For example, the degradation of an organic coating around a metal NP may lead to the exposure of the metal core to the cell interior. As a consequence, the metal cations release could catalyse biochemical reactions or increase intracellular ion concentrations which might negatively affect cell homeostasis. Additionally, NP degradation is directly related to the efficiency of NPs in carrying therapeutics and releasing them at their desired location if they are designed to deliver drugs, and the evaluation of their in vivo stability is critical for the successful translation of drug candidates into the clinic.

The assessment of the in vivo stability of small radiolabelled organic molecules is usually achieved by blood sampling followed by plasma isolation and analysis using instrumental analytical techniques. $^{22,23}$ Although this strategy is also suitable for the assessment of the in vivo stability of radiolabelled NPs, they are not easily isolated from blood samples. Therefore, the problem is often simplified by assessing NP stability in vitro using model media such as water, buffers, or biological fluids ${ }^{24-26}$ and extrapolating the results to in vivo conditions. Despite being valid in some cases, this strategy has severe limitations due to the inherent complexity of biological systems.

The incorporation of multiple imaging agents into a single NP can provide complementary information regarding their stability when used with a combination of imaging modalities. In one of the few reported examples of this approach, oleate-stabilised magnetic NPs with a DSPE-PEG coating were labelled with ${ }^{64} \mathrm{Cu}$ and investigated by combined PET and magnetic resonance imaging (MRI). ${ }^{27}$ However, simultaneous PET-MRI acquisition remains a challenge in the preclinical setting, with consecutive or parallel studies using both modalities required. Additionally, both modalities have very different intrinsic sensitivity, which complicates experimental set up and data analysis. More recently, iron oxide NPs stabilised with oleic acid and phospholipids were radiolabelled with ${ }^{59} \mathrm{Fe},{ }^{14} \mathrm{C}$, and ${ }^{111} \mathrm{In}$ and the biodistribution of all three radioisotopes investigated in mice following sacrifice and organ harvesting. ${ }^{28}$ Although this study generated useful information about NP stability, ${ }^{59} \mathrm{Fe}$ and ${ }^{14} \mathrm{C}$ are inappropriate imaging agents for in vivo studies and animal sacrifice was required.

Very recently, strategies for the preparation of double-labelled functionalised gold NPs have been reported. ${ }^{29,30}$ Following a similar approach, we present here a strategy for the in vivo determination of NP stability, also based on dual radiolabelling using two gamma emitters with different emission energies. In our approach, the NP core and the surface were independently labelled. We exploited the favourable properties of poly(lactide-co-glycolide)
NPs (PLGA-NPs) which, due to their submicron size, display excellent biodegradability and biocompatibility. Additionally, PLGA copolymers were approved by the Food and Drug Administration (FDA) for clinical drug delivery. ${ }^{31}$ Furthermore, their size and the use of emulsion techniques in their preparation allowed our novel approach to be implemented. First, we synthesised iron oxide NPs containing ${ }^{111}$ In and stabilised with oleic acid. Iron oxide NPs were then encapsulated in the PLGA-NP core by mixing them with the PLGA phase during emulsification, overcoming the drawback of chemically modifying PLGA molecules for radiolabelling, which may alter their hydrophobicity and affect NP synthesis. Bovine serum albumin (BSA) was used as a stabilising agent for the emulsion droplets, facilitating the incorporation of ${ }^{125} \mathrm{I}$, the second radioisotope, by electrophilic substitution on the tyrosine residues of the protein. Subsequent SPECT imaging with energy discrimination allowed quantitative assessment of the in vivo stability of NPs, minimising the use of experimental animals. This was achieved by monitoring the change of the intensities of ${ }^{111} \mathrm{In}$ and ${ }^{125} \mathrm{I}$, after appropriate decay correction taking into consideration the half-lives for each isotope. Dissection through gamma counting experiments was conducted and correlated with SPECT images for validation. This general strategy can be extended to a wide variety of engineered NPs and is a valuable tool for in vivo evaluation of NP stability.

\section{Results and discussion}

\section{Synthesis, radiolabelling and characterization of NPs}

The synthesis of dual labelled NPs $\left(\left[{ }^{125} \mathrm{I} /{ }^{111} \mathrm{In}\right]\right.$ NP1, see Fig. 1) consisted of two steps. In the first step, the PLGA core was labelled by including ${ }^{111}$ In-doped iron oxide NPs stabilised with oleic acid during NP fabrication; in the second step, the BSA used as a surface stabiliser was labelled with ${ }^{125} \mathrm{I}$. Radiolabelling of the NP core might be envisioned via attachment of a radiotag to lactic-coglycolic polymers. This process is, however, challenging. Hence, the entrapment of pre-labelled iron oxide NPs was considered as a convenient strategy.

Iron oxide NPs were prepared by a slight modification of a previously published co-precipitation method. ${ }^{32}$ The addition

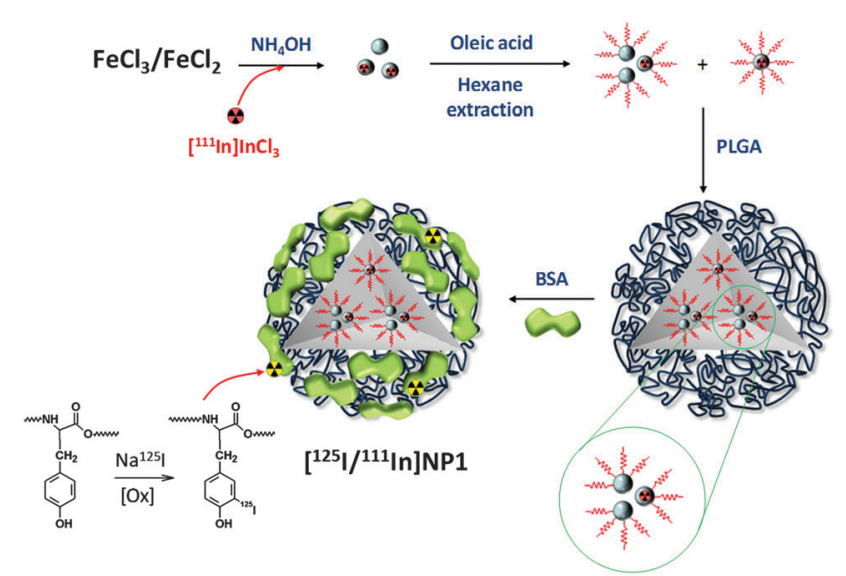

Fig. 1 Schematic of the preparation of dual-labelled $\left[{ }^{125} /{ }^{111} \mid\right.$ n] NP1 NPs. 

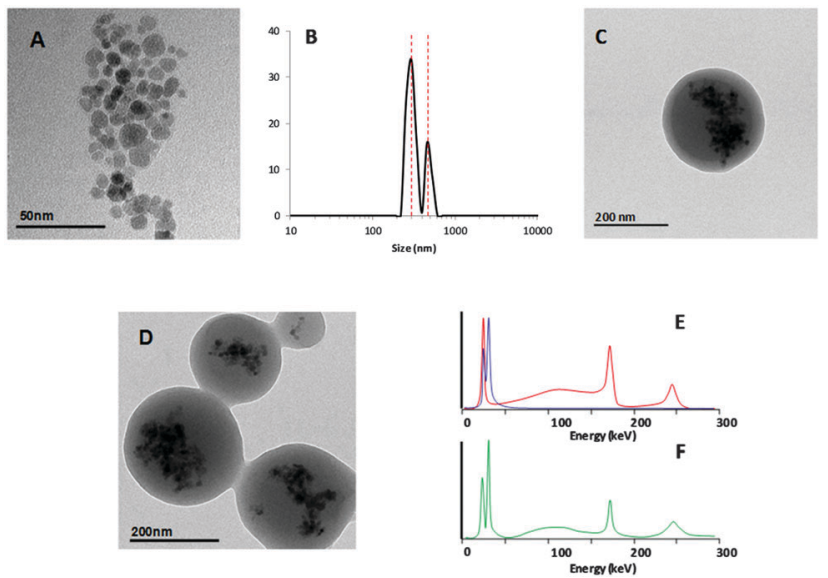

Fig. 2 Characterisation of dual-labelled NPs: (a) TEM images of oleic acid-stabilised iron oxide NPs; (b) intensity distribution for NP1 in water as determined by dynamic light scattering; ( $c$ and d) TEM images of NP1; (e) gamma spectra corresponding to ${ }^{111}$ In (red line) and ${ }^{125}$ I (blue line); (f) gamma spectrum of NP1.

of ${ }^{111} \mathrm{InCl}_{3}$ during co-precipitation yielded mono-disperse iron oxide magnetic NPs coated with oleic acid, with a diameter of $12 \pm 3 \mathrm{~nm}$ as determined by TEM (Fig. 2a). NPs were further dispersed in an organic PLGA solution using a well-established protocol. ${ }^{33}$ An aqueous BSA solution was transferred to the organic phase, and the solution was sonicated to form organic phase droplets stabilised with BSA and containing iron oxideloaded PLGA-NPs ([111 In]NP1). Finally, the incorporation of ${ }^{125} \mathrm{I}$ by electrophilic aromatic substitution was carried out to yield $\left[{ }^{125} \mathrm{I} /{ }^{111} \mathrm{In}\right] \mathbf{N P 1}$.

The selection of the correct combination of radioisotopes was of paramount importance to enable subsequent imaging studies using energy discriminant SPECT. Ideally, the energy spectra should not overlap, and the energy peaks should fall within the energy range of the SPECT system used (in our case 25-250 keV). Additionally, both radioisotopes should have a sufficient half-life to investigate biodistribution for long periods of time after administration. ${ }^{111}$ In $\left(T_{1 / 2}=2.8\right.$ days, maximum energy emission at 171 and $245 \mathrm{keV}$ ) is a radioisotope with good in vivo imaging characteristics, which has been widely used in both the pre-clinical and clinical settings. It is usually attached to the molecule of interest by the formation of a radiometalchelator complex; however, previous studies have suggested that it can be readily incorporated into the crystal lattice of iron oxide $\mathrm{NPs},{ }^{34}$ as here. For BSA labelling, ${ }^{125} \mathrm{I}$ was chosen for several reasons: first, it has been widely used for the radiolabelling of peptides, proteins, and antibodies via electrophilic substitution by taking advantage of the presence of tyrosine residues; second, ${ }^{125} \mathrm{I}$ has an energy maximum at $35.5 \mathrm{keV}$ and a long half-life (close to 60 days) and is reasonably cheap and widely available. Hence, both isotopes have a relatively long half-life, appropriate emission characteristics, and established radiolabelling routes.

The hydrodynamic diameter of $\left[{ }^{125} \mathrm{I} /{ }^{111} \mathrm{In}\right] \mathbf{N P 1}$ NPs was determined by dynamic light scattering (DLS): the mean NP size was $300 \pm 40 \mathrm{~nm}(\sim 75 \%)$, with a minor population $(\sim 25 \%)$ of $460 \pm 70 \mathrm{~nm}$ (Fig. 2b) also observed in the intensity distribution.
TEM analysis performed after radioactive decay showed that iron oxide NPs were properly entrapped in the polymeric matrix core (Fig. 2c and d). TEM imaging under dry conditions revealed PLGA-NP sizes of about $250 \pm 50 \mathrm{~nm}$. The PLGA-NPs had a negative $\xi$-potential of $-15 \mathrm{mV}$ at neutral $\mathrm{pH}$. Gamma spectrometry of the final NPs showed the presence of peaks corresponding to ${ }^{111} \mathrm{In}$ and ${ }^{125} \mathrm{I}$ (Fig. 2e and f). The ratio of both isotopes was always maintained in the range ${ }^{125} \mathrm{I} /{ }^{111} \mathrm{In}=1.3-1.6$.

The stability of $\left[{ }^{125} \mathrm{I} /{ }^{111} \mathrm{In}\right] \mathbf{N P 1}$ was measured in physiological saline $(0.9 \% \mathrm{NaCl})$ and mouse plasma at $37{ }^{\circ} \mathrm{C}$. The NPs showed good stability in physiological saline (Fig. 3), with both isotopes remaining attached to the NPs up to 48 hours. At $t=6$ days, significant release of ${ }^{125}$ I and ${ }^{111}$ In was observed, although $64 \%$ and $75 \%$ of the radioactivity, respectively, remained attached to the NPs.

Interestingly, incubation in plasma resulted in the progressive release of ${ }^{125} \mathrm{I}$. At $48 \mathrm{~h}$ of incubation, approximately $50 \%$ of the ${ }^{125}$ I was released from the NPs. This value increased up to $85 \%$ at $t=6$ days. The decrease in the ${ }^{125}$ I signal observed in mouse serum hints at the removal of labelled BSA from the NPs. BSA can be replaced by unlabelled proteins from the serum or simply released as a consequence of their interaction with serum components. BSA molecules are linked to the PLGA only by hydrophobic interactions, which may not be strong enough to guarantee their attachment to the NP surface in the presence of serum components. On the other hand, the ${ }^{111}$ In percentage remained almost constant up to 2 days, with a progressive decrease afterwards to reach values around $65 \%$ at $t=6$ days.
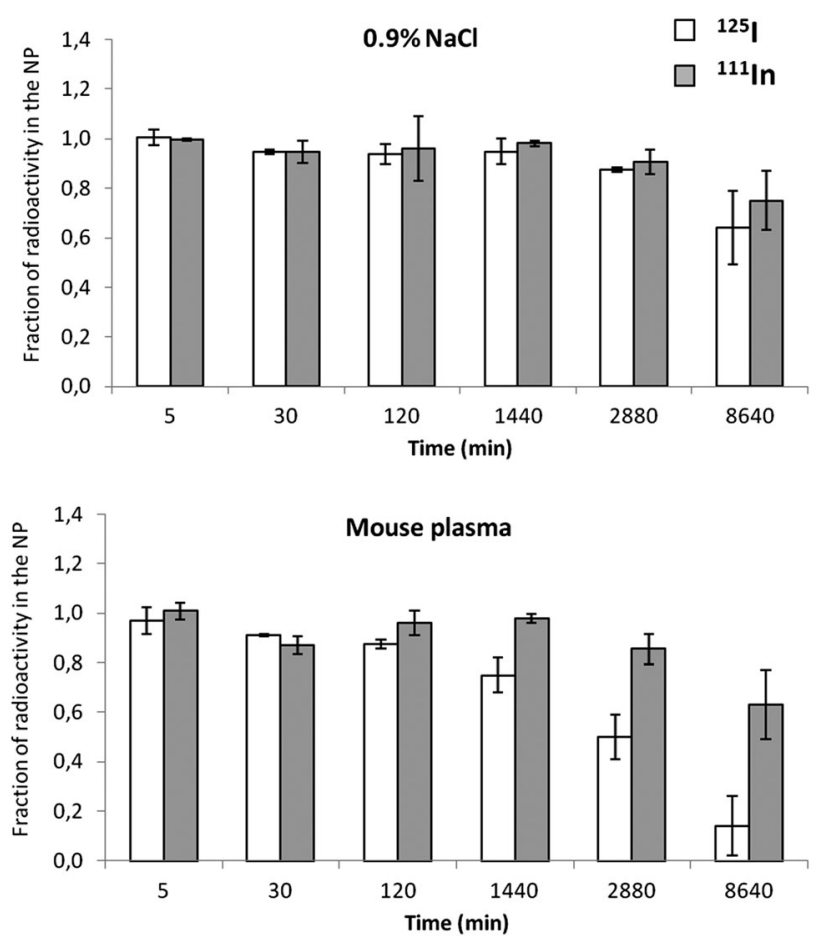

Fig. 3 Stability of $\left[{ }^{125} /{ }^{111}\right.$ In]NP1 in physiological saline (top) and mouse plasma (bottom) expressed as \% radioactivity attached to the NP at different time points of incubation $\left(T=37^{\circ} \mathrm{C}\right)$. Results are expressed as mean \pm standard deviation ( $n=3$ per time point). 
These values suggest that iron oxide NPs remain PLGA encapsulated over the first $48 \mathrm{~h}$ of the experiment, and the release occurs very slowly and only at long times after incubation. This was expected, since degradation (weight loss) of PLGA in vitro is reported to only occur after at least one week in phosphatebuffered saline ( $\mathrm{pH} 7.4){ }^{35,36}$

\section{In vivo imaging studies}

Stability studies were followed by in vivo SPECT imaging; static images were acquired for each radioisotope at different time points after administration using a SPECT camera with energy discrimination (Fig. 4a); pure ${ }^{125}$ I-labelled BSA was used as a control (Fig. 4b).

SPECT imaging after administration of the NPs showed that the biodistribution of the two radiotags could be followed independently. Three hours after administration, the majority of the radioactivity of both radioisotopes (red for ${ }^{125} \mathrm{I}$, green for ${ }^{111} \mathrm{In}$ ) was located in the liver and lungs with ${ }^{125} \mathrm{I} /{ }^{111} \mathrm{In}$ ratios of $1.12 \pm 0.21$ and $0.92 \pm 0.32$, respectively (Fig. 4a), suggesting that the majority of the NPs remained intact in these organs. Accumulation in the lung was probably due to the size of the NPs, which may form micro-emboli by occlusion of the small lung capillaries. $^{37}$ Sequestration by the reticulo-endothelial system (RES) may also contribute to lung accumulation. At this time point, there was selective accumulation of ${ }^{125} \mathrm{I}$ in the bladder
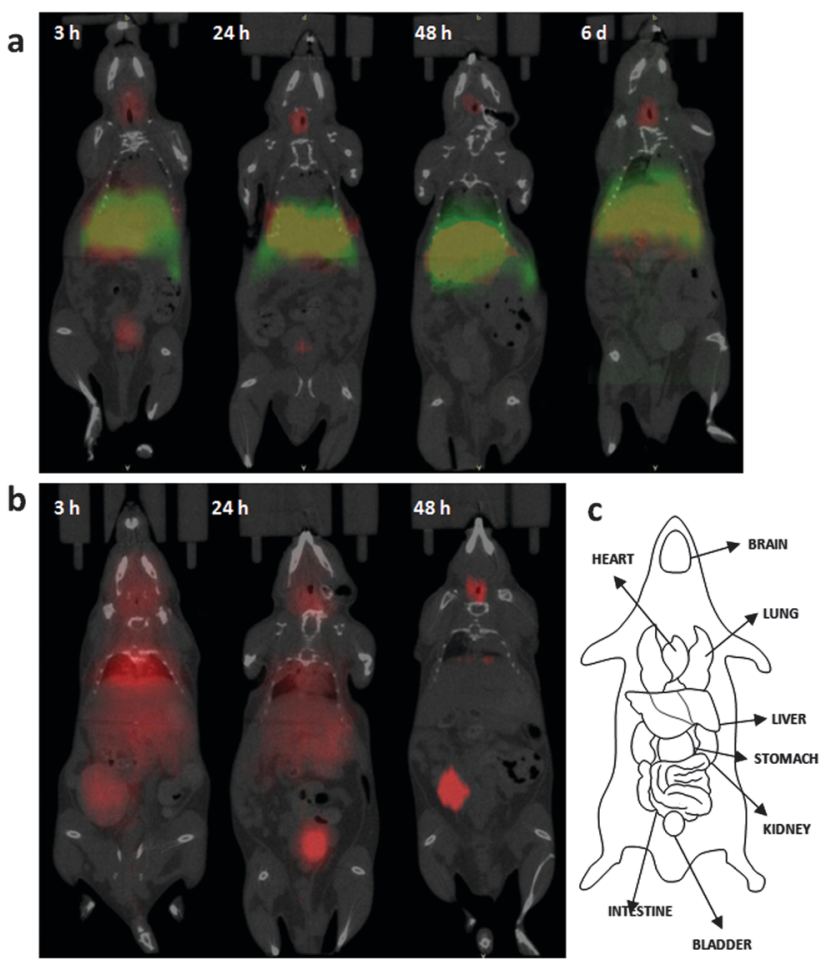

Fig. 4 Coronal SPECT images of the biodistribution of (a) $\left[{ }^{125} / /{ }^{111} \mid n\right] N P 1$ and (b) $\left[{ }^{125}\right.$ I]BSA after intravenous administration into mice; red colour: ${ }^{125}$; green colour: ${ }^{111} \mathrm{In}$. Images were co-registered with a CT image of the same animal to anatomically localize the SPECT signal. CT images were adjusted in the $Y$ axis at each time point for appropriate fitting with the tracer distribution image. (c) A schematic of the different organs. and thyroid glands, suggesting that the NPs had already undergone some degradation. One and two days after administration, a fraction of ${ }^{125} \mathrm{I}$ and ${ }^{111} \mathrm{In}$ still co-localised in the liver and lungs $\left({ }^{125} \mathrm{I} /{ }^{111} \mathrm{In}\right.$ ratio $=1.07 \pm 0.14$ and $0.92 \pm 0.18$ in the liver at $t=24$ and $48 \mathrm{~h}$, respectively; ${ }^{125} \mathrm{I} /{ }^{111} \mathrm{In}$ ratio $=0.67 \pm 0.22$ and $0.42 \pm 0.16$ in the lungs at $t=24$ and $48 \mathrm{~h}$, respectively), but the ${ }^{125}$ I signal in the thyroid gland progressively increased with time. Six days after NP administration, ${ }^{125}$ I was almost cleared from the body (except the thyroid gland), with the majority of detected radioactivity originating from ${ }^{111}$ In in the liver. This suggests that the NP core had not fully degraded.

In order to obtain comparative data, pure ${ }^{125}$ I-labelled BSA was also administered to the animals and SPECT scans were conducted at three, 24, and 48 hours (Fig. 4b). Pure ${ }^{125}$ I-labelled BSA rapidly accumulated in the lungs, liver, thyroid gland, and urine. ${ }^{125} \mathrm{I}$ was detected in the liver after $24 \mathrm{~h}$ and was almost cleared from this organ by $48 \mathrm{~h}$, at which point radioactivity was only detected in the bladder and thyroid gland.

Altogether, the results suggest that $\left[{ }^{125} \mathrm{I} /{ }^{111} \mathrm{In}\right] \mathbf{N P 1}$ biodistributes due to the attachment of the protein to the NPs, and at initial time points the BSA follows the biodistribution of the core. It has previously been reported that ${ }^{125} \mathrm{I}$-labelled BSA undergoes rapid clearance from mouse livers after intravenous administration $(<0.2 \%$ of injected dose after 24$)$, with the labelled metabolite $\left[{ }^{125} \mathrm{I}\right] \mathrm{mono}\left(\mathrm{di}\right.$-)iodotyrosin detected in hepatocytes. ${ }^{38}$ This radiometabolite can easily cross biological membranes, which might explain the low uptake at later time points. The elimination of ${ }^{125}$ I was much less dramatic from dual labelled NPs $\left({ }^{125} \mathrm{I}\right.$ remained detectable in the liver six days after administration), suggesting that the radiolabelled BSA remained attached to NPs in the liver.

\section{Ex vivo studies}

The biodistribution and chemical stability of the NPs were also investigated by dissection and gamma counting, which also provided accurate, organ-level biodistribution information and corroborated the in vivo data. The accumulation of ${ }^{125} \mathrm{I}$ and ${ }^{111}$ In in different organs expressed as the \% of injected dose per gram of tissue (\%ID $\mathrm{g}^{-1}$ ) is shown in Fig. 5.

For both radioisotopes, the highest radioactivity concentrations were detected in the lungs at early time points after administration (\%ID $\mathrm{g}^{-1} \sim 40$ at $t<2 \mathrm{~h}$ ). The liver had the next highest concentrations (\%ID g ${ }^{-1}$ between 20 and $30 \%$ ), with a non-significant increasing trend observed between 5 and $30 \mathrm{~min}$ and a progressive decrease thereafter. After $48 \mathrm{~h}$, the $\% \mathrm{ID} \mathrm{g} \mathrm{g}^{-1}$ were $11.6 \pm 3.2$ and $3.3 \pm 1.8$ for ${ }^{125} \mathrm{I}$ and $16.6 \pm 4.6$ and $9.6 \pm 3.2$ for ${ }^{111}$ In in the liver and lungs, respectively. The accumulation of ${ }^{125} \mathrm{I}$ in the thyroid gland increased over time, reaching values close to $100 \% \mathrm{ID} \mathrm{g}^{-1}$ at $48 \mathrm{~h}$. Interestingly, the accumulation of ${ }^{111}$ In in the thyroid glands was very low $(<3 \%)$ at all time points, which can be considered insignificant given the very small size of the thyroid gland (between 4-5 $\mathrm{mg}$ ). The accumulation of ${ }^{125}$ I was extremely high in the urine soon after administration $\left(131 \pm 33 \% \mathrm{ID} \mathrm{g}^{-1}\right.$ at $\left.t=30 \mathrm{~min}\right)$, with accumulation of ${ }^{111}$ In below 5\% throughout the experiment. Values below $0.1 \% \mathrm{ID} \mathrm{g}^{-1}$ were obtained for both radioisotopes 

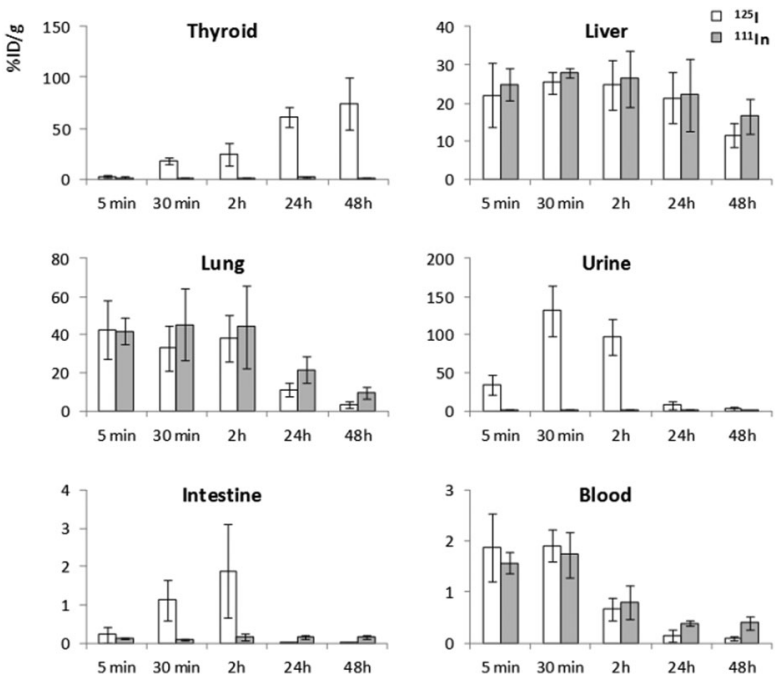

Fig. 5 Accumulation of ${ }^{125} \mathrm{I}$ and ${ }^{111}$ In in different organs as determined by dissection and gamma counting and expressed as the \% of injected dose per gram of tissue $\left(\% \mathrm{ID} \mathrm{g}^{-1}\right)$. Results are expressed as mean \pm standard deviation ( $n=3$ per time point).

in the brain (results not shown). Blood radioactivity was similar for both isotopes, with initial $\%$ ID $\mathrm{g}^{-1}$ values in the range of $1.5-2 \%$, which progressively decreased to below $1 \%$ at $t>2 \mathrm{~h}$.

${ }^{125} \mathrm{I} /{ }^{111} \mathrm{In} \% \mathrm{ID} \mathrm{g}{ }^{-1}$ ratios at different time points for different organs after intravenous administration of the dual-labelled NPs are shown in Fig. 6: values close to 1 denote that the amounts of both isotopes (expressed as $\% \mathrm{ID} \mathrm{g}^{-1}$ ) are equivalent, suggesting that the two labels remain together and that the NPs have not degraded. At early time points after administration, the ${ }^{125} \mathrm{I} /{ }^{111} \mathrm{In}$ ratio was always close to 1 , irrespective of the organ analysed. This ratio was maintained in the liver up to $24 \mathrm{~h}$, with a slight, non-significant decrease thereafter $(0.70 \pm 0.27$ at $t=48 \mathrm{~h})$. A similar trend was observed in the lungs, with values very close
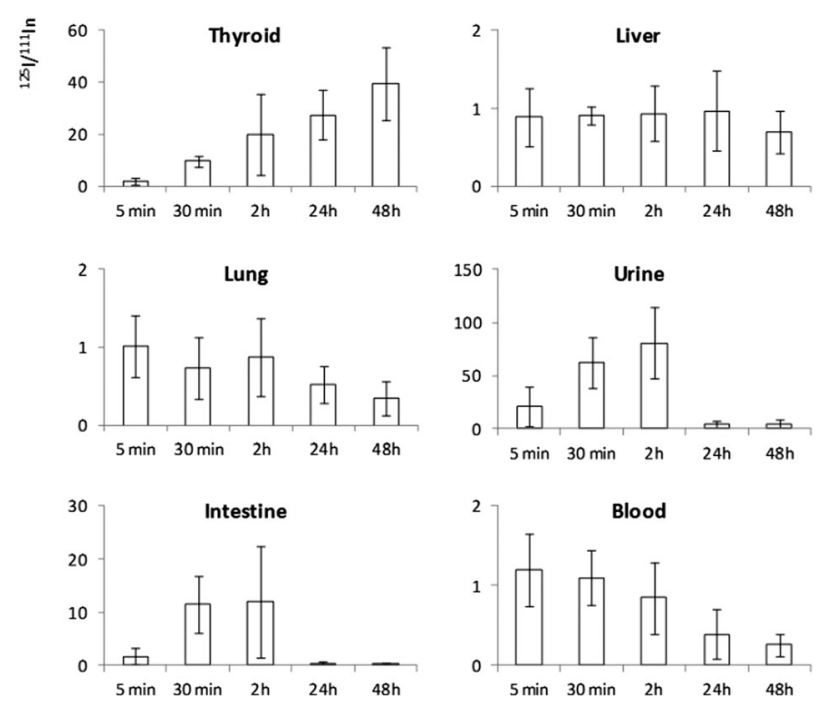

Fig. ${ }^{125} / /^{111}$ In ratios in different organs as determined by dissection and gamma counting. Results are expressed as mean \pm standard deviation ( $n=3$ per time point). to 1 at early time points after administration. However, a decreasing trend was readily observed by $24 \mathrm{~h}(0.52 \pm 0.24$ at $t=24 \mathrm{~h})$ that became more pronounced at $t=48 \mathrm{~h}(0.34 \pm 0.22)$. These values are concordant with those obtained in vivo using SPECT and energy discrimination. The ${ }^{125} \mathrm{I} /{ }^{111} \mathrm{In}$ ratios in the urine, intestine, and thyroid glands were very high even at early time points after NP administration, confirming selective accumulation of radioiodine in these tissues. As observed in the in vitro stability studies and in vivo SPECT experiments, these results suggest that labelled proteins slowly detached from the NP core in the presence of plasma proteins and this phenomenon was likely to have been even quicker in the in vivo circulation.

The abovementioned degradation of the protein coating which might have been replaced by other proteins during circulation was previously reported in in vitro studies, ${ }^{39}$ and is an important issue since it can alter the biodistribution of NPs. Most coatings are not only used to protect the NP core or stabilise them in aqueous media, but also to direct the NPs to specific organs or tissues or to carry specific functions. The loss of these functionalities may lead to modification of the properties of NPs. In the case considered here, BSA is not covalently bonded to the NPs but interacts with PLGA by hydrophobic interactions, since it was used as a surfactant to stabilise the PLGA emulsion that led to the formation of the NPs. Under these conditions, the NP-PLGA core interaction is weak, which probably makes the removal of BSA easy. Covalently attaching the protein to the NP would most likely increase protein stability. This knowledge about the stability of the BSA coating is helpful for the design of surface coatings of NPs and how to engineer them to produce longer circulation times or improve their stability.

\section{Experimental section}

\section{Reagents}

All chemical reagents and solvents were purchased from SigmaAldrich (St. Louis, MO, USA) and were of analytical grade purity unless otherwise specified. Nanopure water $(18.2 \mathrm{M} \Omega \mathrm{cm})$ was obtained using a Thermo Scientific Barnstead NANOpure Diamond Water System (Thermo Fisher Scientific, Waltham, MA, USA). Na $\left.{ }^{125} \mathrm{I}\right] \mathrm{I}$ (solution in $0.1 \mathrm{M} \mathrm{NaOH}$ ) was obtained from Perkin Elmer (Beaconsfield, UK), and ${ }^{111} \mathrm{InCl}_{3}$ (solution in $\mathrm{HCl}$ ) was purchased from Covidien (Dublin, Ireland).

\section{Preparation of ${ }^{111}$ In-labelled iron oxide NPs}

Iron(II) chloride tetrahydrate $(50 \mathrm{mg}$ ) and iron(III) chloride hexahydrate $(60 \mathrm{mg})$ were dissolved in ultrapure water $(25 \mathrm{~mL})$. A fraction of this solution $(0.5 \mathrm{~mL})$ was withdrawn using a pipette and diluted with ultrapure water $(4.5 \mathrm{~mL})$ in a vial. ${ }^{111} \mathrm{InCl}_{3}$ solution $(780 \mu \mathrm{L}, 512 \mathrm{MBq})$ was immediately added, and the vial was capped and flushed with nitrogen gas (solution A). In parallel, ammonia ( $29 \%$ aqueous solution, $2.34 \mathrm{~mL}$ ) was added to a $100 \mathrm{~mL}$ flask and diluted with ultrapure water to a final volume of $20 \mathrm{~mL}$. The solution was stirred for $30 \mathrm{~min}$ under nitrogen gas flow (solution B). Solution A was then slowly 
added to solution B with constant stirring (500 rpm) at room temperature. The solution turned yellow-black due to the formation of iron oxide NPs. After $30 \mathrm{~min}$, the NPs were collected using a magnet, washed with water three times, and finally dispersed in purified water $(5 \mathrm{~mL})$.

\section{Preparation of oleic acid-modified ${ }^{111}$ In-labelled iron oxide NPs}

Radiolabelled iron oxide NPs prepared as above were sonicated ( $20 \%$ power, $2 \mathrm{~s}+2 \mathrm{~s}$ cycles) for $30 \mathrm{~min}$ (total running time). Oleic acid $(100 \mu \mathrm{L})$ was then added dropwise under sonication. To prevent a temperature rise in the reaction mixture, a water bath $\left(20^{\circ} \mathrm{C}\right)$ was used. After $2 \mathrm{~h}$, the NPs were collected using a magnet. To recover NPs attached to the sonication tip and to the walls of the vial, the aqueous solution was removed by decantation, hexane $(1 \mathrm{~mL})$ was added, and the mixture was further sonicated. Finally, the collected NPs were dispersed into hexane and the solution was concentrated to a final volume of $25 \mu \mathrm{L}$.

\section{Preparation of PLGA-BSA particles}

Oleic acid-modified ${ }^{111}$ In-labelled iron oxide NPs as prepared above $(25 \mu \mathrm{L})$ were added to a PLGA (PLGA 85:15) solution ( $2 \%$ in dichloromethane, $125 \mu \mathrm{L}$ ) and the resulting mixture was sonicated for $10 \mathrm{~min}$. BSA solution ( $2 \%$ in purified water, $600 \mu \mathrm{L}$ ) was added, and the mixture was again sonicated for 20 s. The resulting solution was immediately poured into purified water $(15 \mathrm{~mL})$ and stirred for $2 \mathrm{~h}$. PLGA NPs were then collected by centrifugation and washed twice with purified water. The resulting NPs were finally dispersed in PBS $(50 \mu \mathrm{L})$.

\section{Radiolabelling of PLGA-BSA NPs with ${ }^{125}$ I}

Iodination was carried out using Pierce ${ }^{\circledR}$ iodination beads. The beads were washed with PBS $(1 \mathrm{~mL})$, dried with filter paper, and introduced to a vial with PBS $(100 \mu \mathrm{L}, \mathrm{pH}=7.2)$ and $\mathrm{Na}^{125} \mathrm{I}$ (ca. $20 \mu \mathrm{L}, 37 \mathrm{MBq}$ ). The mixture was incubated at room temperature for $5 \mathrm{~min}$ with occasional vigorous shaking. PLGA-BSA NPs (PBS solution, see above) were added to this solution, and the mixture was incubated for $15 \mathrm{~min}$ at room temperature. The NPs were finally isolated by centrifugation and washed twice with PBS.

\section{NP characterisation}

Size distributions and $\xi$-potentials were determined for all samples. Transmission electron microscopy (TEM) was performed using a JEOL JEM-1400 plus microscope (Jeol, Tokyo, Japan) working at $120 \mathrm{kV}$. For TEM examinations, a single drop $(10 \mu \mathrm{L})$ of the aqueous solution ( $\sim 0.1 \mathrm{mg} \mathrm{mL}{ }^{-1}$ in milliQ water) of the NPs was placed onto a carbon film on 400 square mesh copper grids (CF-400-Cu, Electron Microscopy Sciences, Hatfield, PA, USA). The grid was left to dry in air for several hours at room temperature.

DLS and $\xi$-potential measurements were performed using a Malvern Zetasizer Nano ZS system (Malvern Instruments, Malvern, UK). For particle size determination, ten measurements for $10 \mathrm{~s}$ were carried out at a temperature of $25{ }^{\circ} \mathrm{C}$ at a $173^{\circ}$ scattering angle. A refractive index of 1.52 for PLGA was used. For $\xi$-potential measurements, a minimum of 15 runs were carried out. NP effective surface charge in $10 \mathrm{mM}$ PBS was measured at $\mathrm{pH}$ 7.4. Measurements were performed in quintuplicate. The Smoluchowski approximation was used to convert the electrophoretic mobility to $\xi$-potential values.

Radiolabelled NPs were also characterised by $\gamma$-spectrometry using a multi-channel analyser $\gamma$-spectrometer (MUCHA, Raytest, Straubenhardt, Germany) calibrated with standard energy calibration sources. A small fraction of the NPs was placed into a vial and the energy spectrum was recorded. The areas under the peaks at $35 \mathrm{kV}$ (maximum emission for ${ }^{125} \mathrm{I}$ ) and $171 \mathrm{keV}$ (energy peak for ${ }^{111} \mathrm{In}$ ) were used to determine the absolute amounts of both radioisotopes.

\section{Radiochemical integrity of labelled NPs}

To assess the radiochemical stability, NPs were prepared and purified as described above and subsequently resuspended in $300 \mu \mathrm{L}$ of PBS or mouse plasma. The suspension was then divided into five different aliquots containing $50 \mu \mathrm{L}$ of the NPS each. The aliquots were kept at $37{ }^{\circ} \mathrm{C}$ for five and $30 \mathrm{~min}$, and two, 24, 48 and 144 hours, respectively. The samples were then centrifuged and the NPs were separated by decantation and washed twice with PBS. The energy spectra of the supernatant and the NPs were then recorded, and the absolute amount of ${ }^{125} \mathrm{I}$ and ${ }^{111} \mathrm{In}$ in both fractions was determined.

\section{Animal experiments}

Animals. All the animal procedures were performed in accordance with the Spanish policy for animal protection (RD53/2013), which meets the requirements of the European Union directive 2010/63/UE regarding the protection of animals used in experimental procedures. The guidelines were approved by the Ethical Committee of CIC biomaGUNE and authorised by the regional government. Mice (BALB/cJRJ, 8-12 weeks of age, Janvier Labs, France) were housed in a controlled environment (12:12 light/dark cycle with dawn and dusk transitional periods, room temperature $22{ }^{\circ} \mathrm{C}$, and $55 \%$ relative humidity) and maintained on commercially available pelleted diet and sterilised water ad libitum. Three animals per labelled species were used for in vivo SPECT/CT experiments; dissection and gamma counting experiments were performed on three animals at each time point.

SPECT/CT images. SPECT/CT images were acquired using the eXplore speCZT CT preclinical imaging system (GE Healthcare, Little Chalfont, UK). This system uses a stationary, full ring of cadmium zinc telluride (CZT) detectors and interchangeable rotating cylindrical collimators. The CZT detectors offer substantially better energy and spatial resolution than the NaI detectors used in conventional SPECT systems. The improved energy resolution is particularly important for applications such as dual isotope imaging since it allows for better energy peak separation and rejection of scattered photons to yield higher contrast images.

Three hours prior to examination, mice (three per labelled species) received an intravenous administration of either (i) $\left[{ }^{125} \mathrm{I} /{ }^{111} \mathrm{In}\right] \mathbf{N P 1}$ or (ii) ${ }^{125}$ I-labelled BSA. With the mouse under isoflurane anaesthesia (1.5-2\% in oxygen), whole-body SPECT/ CT scans were acquired at $3 \mathrm{~h}, 24 \mathrm{~h}, 48 \mathrm{~h}$, and 6 days postinjection (p.i.). Using a full ring detector, $360^{\circ}$ of data were acquired by rotating the collimator at $45^{\circ}$ ( 45 steps, $1^{\circ}$ per step). 
Data were collected in an energy window of $30-200 \mathrm{keV}$, and the duration of the scans ranged between two and four hours. As a general rule, the image acquisition time was chosen based on the number of counts detected; thus, longer imaging times were required for later p.i. scans. During image acquisition, mice were kept under normothermic conditions using a heating blanket (Homeothermic Blanket Control Unit; Bruker BioSpin $\mathrm{GmbH}$, Karlsruhe, Germany). After each SPECT scan, CT acquisitions were performed to provide anatomical information about each animal. The CT acquisition consisted of 220 views acquired in $0.88^{\circ}$ increments around the animal with $16 \mathrm{~ms}$ exposure per view. The X-ray tube settings were $70 \mathrm{kV}$ and $32 \mathrm{~mA}$.

The SPECT images were reconstructed using the 2D OSEM iterative algorithm with 10 iterations/1 subsets for ${ }^{111}$ In in the 154-188 keV energy window, and 5 iterations/1 subsets for ${ }^{125} \mathrm{I}$ in the $27-35 \mathrm{keV}$ energy window. The images were reconstructed into $128 \times 128 \times 32$ arrays with a voxel size of $0.4 \times 0.4 \times 2.46 \mathrm{~mm}$ and were corrected for scatter but not attenuation. The CT images were reconstructed using a cone beam filtered back-projection Feldkamp algorithm into $437 \times$ $437 \times 523$ arrays with a voxel size of $0.2 \times 0.2 \times 0.2 \mathrm{~mm}$.

Dissection and gamma counting: animals $(n=30)$ were anesthetised with isofluorane and a solution of dual-labelled NPs (containing ca. $0.8 \mathrm{MBq}$ of ${ }^{125} \mathrm{I}$ and $0.5 \mathrm{MBq}$ of ${ }^{111} \mathrm{In}$ ) injected through the tail vein. After injections, animals were allowed to recover. Just before sacrifice by cervical dislocation (five and $30 \mathrm{~min}$ and two, 24, and 48 after dose administration), animals were anesthetised again and blood samples were collected by cardiac puncture. After sacrifice, liver, lungs, brain, cerebellum, intestine, and thyroid glands were quickly removed and rinsed twice with deionised water; urine samples were also obtained. The gamma spectra for organs and fluids were obtained in the multi-channel analyser $\gamma$-spectrometer (MUCHA, Raytest).

\section{Conclusions}

In conclusion, we presented a novel methodology for the synthesis of dual-radiolabelled BSA-coated PLGA-NPs with two gamma emitters without overlapping energy spectra located in the core and at the surface. The biodistribution and biological fate of the labels at the surface coating and in the core could be determined independently by SPECT imaging and corroborated by dissection and gamma counting. Imaging showed that NPs accumulated in the lungs and liver at early time points, at which point the ${ }^{111} \mathrm{In} /{ }^{125} \mathrm{I}$ ratio remained constant; however, ${ }^{125}$ I progressively accumulated in the thyroid glands and urine, resulting in a decrease in co-localisation with the signal corresponding to ${ }^{111}$ In located at the core. After six days, there was almost complete clearance of ${ }^{125} \mathrm{I}$, but the ${ }^{111}$ In signal was still detectable in the liver. These results indicate that ${ }^{125}$ I-labelled BSA is progressively removed from the core to follow a different pattern of biodistribution and clearance than the core. These results help in explaining the fate and process of degradation of the surface coatings of PLGA-NPs, and this methodology represents an excellent alternative for visualising the degradation process of multi-labelled NPs in vivo.

\section{Acknowledgements}

The authors would like to thank the Ministerio de Economía y Competitividad (MINECO, project MAT2013-48169-R), the Departamento de Educación, Política Lingüística y Cultura del Gobierno Vasco (project PI-2014-1-90), the FP7-Infrastructures project QNANO (Project reference: 262163), and the Natural Science Foundation of China (51120135001) for financial support. The authors also would like to thank Eleftheria Diamanti for assistance in Figure design.

\section{Notes and references}

1 A. Lankoff, W. J. Sandberg, A. Wegierek-Ciuk, H. Lisowska, M. Refsnes, B. Sartowska, P. E. Schwarze, S. Meczynska-Wielgosz, M. Wojewodzka and M. Kruszewski, Toxicol. Lett., 2012, 208, 197-213.

2 S. Parveen, R. Misra and S. K. Sahoo, Nanomed.: Nanotechnol. Biol. Med., 2012, 8, 147-166.

3 W. H. De Jong, M. C. Burger, M. A. Verheijen and R. E. Geertsma, Materials, 2010, 3, 4681-4694.

4 J. Moger, B. D. Johnston and C. R. Tyler, Opt. Express, 2008, 16, 3408-3419.

5 X. Zhou, M. Dorn, J. Vogt, D. Spemann, W. Yu, Z. Mao, I. Estrela-Lopis, E. Donath and C. Gao, Nanoscale, 2014, 6, 8535-8542.

6 W. H. De Jong, W. I. Hagens, P. Krystek, M. C. Burger, A. J. A. M. Sip and R. E. Geertsma, Biomaterials, 2008, 29, 1912-1919.

7 K. Stockhofe, J. M. Postema, H. Schieferstein and T. L. Ross, Pharmaceuticals, 2014, 7, 392-418 (and references therein).

8 C. Pérez-Campaña, V. Gómez-Vallejo, M. Puigivila, A. Martín, T. Calvo-Fernández, S. E. Moya, R. F. Ziolo, T. Reese and J. Llop, ACS Nano, 2013, 7, 3498-3505.

9 K. Abbas, I. Cydzik, R. del Torchio, M. Farina, E. Forti, N. Gibson, U. Holzwarth, F. Simonelli and W. Kreyling, J. Nanopart. Res., 2010, 12, 2435-2443.

10 F. Simonelli, P. Marmorato, K. Abbas, J. Ponti, J. Kozempel, U. Holzwarth, F. Franchini and F. Rossi, IEEE Trans. NanoBiosci., 2011, 10, 44-50.

11 S. H. Jung, K. I. Kim, J. H. Ryu, S. H. Choi, J. B. Kim, J. H. Moon and J. H. Jin, Appl. Radiat. Isot., 2010, 68, 1025-1029.

12 J. Roy and S. Lahiri, Green Chem., 2006, 8, 1063-1066.

13 S. Hirn, M. Semmler-Behnke, C. Schleh, A. Wenk, J. Lipka, M. Schaffler, S. Takenaka, W. Moller, G. Schmid, U. Simon and W. G. Kreyling, Eur. J. Pharm. Biopharm., 2011, 77, 407-416.

14 J. Frigell, I. Garcia, V. Gómez-Vallejo, J. Llop and S. Penades, J. Am. Chem. Soc., 2014, 136, 449-457.

15 B. R. Jarrett, B. Gustafsson, D. L. Kukis and A. Y. Louie, Bioconjugate Chem., 2008, 19, 1496-1504.

16 E. J. Keliher, J. Yoo, M. Nahrendorf, J. Lewis, B. Marinelli, A. Newton, M. Pittet and R. Weissleder, Bioconjugate Chem., 2011, 22, 2383-2389. 
17 S. Guerrero, J. R. Herance, S. Rojas, J. F. Mena, J. D. Gispert, G. A. Acosta, F. Albericio and M. J. Kogan, Bioconjugate Chem., 2012, 23, 399-408.

18 S. Rojas, J. D. Gispert, R. Martín, S. Abad, C. Menchón, D. Pareto, M. V. Víctor, M. álvaro, H. García and J. R. Herance, ACS Nano, 2011, 5, 5552-5559.

19 J. J. Willman, Z. Cheng, C. Davis, A. M. Lutz, M. L. Schipper, C. H. Nielsen and S. S. Gambhir, Radiology, 2006, 249, 212-219.

20 P. P. Di Mauro, V. Gómez-Vallejo, Z. Baz, J. Llop and S. Borros, Bioconjugate Chem., 2015, 26, 582-592.

21 S. J. Soenen, J. M. Montenegro, A. M. Abdelmonem, B. B. Manshian, S. H. Doak, W. J. Parak, S. C. de smedt and K. Braeckmans, Acta Biomater., 2014, 10, 732-741.

22 I. Odano, C. Halldin, P. Karlsson, A. Varrone, A. J. Airaksinen and R. N. Farde, NeuroImage, 2009, 45, 891-902.

23 V. Gómez-Vallejo, A. Martín, M. Aginalde, E. San Sebastián, D. Padro, F. Cossio and J. Llop, Appl. Radiat. Isot., 2012, 70, 2545-2551.

24 R. Sharma, Y. Xu, S. W. Kim, M. J. Schueller, D. Alexoff, S. D. Smith, W. Wang and D. Schlyer, Nanoscale, 2013, 5, 7476-7483.

25 A. Polyák, I. Hajdu, M. Bodnan, G. Trencsenyi, Z. Postenyi, V. Haasz, G. Janoki, L. Balogh and J. Borbely, Int. J. Pharm., 2013, 449, 10-17.

26 B. E. Ocampo-García, F. de M. Ramírez, G. Ferro-Flores, L. M. de León-Rodríguez, C. L. Santos-Cuevas, E. Morales-ávila, C. Arteaga de Murphy, M. Pedraza-López, L. A. Medina and M. A. Camacho-López, Nucl. Med. Biol., 2010, 38, 1-11.

27 C. Glaus, R. Rossin, M. J. Welch and G. Bao, Bioconjugate Chem., 2010, 21, 715-722.
28 H. Wang, R. Kumar, D. Nagesha, R. I. Duclos Jr, S. Sridhar and S. J. Gatley, Nucl. Med. Biol., 2015, 42, 65-70.

29 K. C. L. Black, W. J. Akers, G. Sudlow, B. Xu, R. Laforest and S. Achilefu, Nanoscale, 2015, 7, 440-444.

30 W. G. Kreyling, A. M. Abdelmonem, Z. Ali, F. Alves, M. Geiser, N. Haberl, R. Hartmann, S. Hirn, D. Jimenez de Aberasturi, K. Kantner, G. Khadem-Saba, J.-M. Montenegro, J. Rejman, T. Rojo, I. Ruiz de Larramendi, R. Ufartes, A. Wenk and W. J. Parak, Nat. Nanotechnol., 2015, DOI: 10.1038/nnano.2015.111.

31 J. Nicolas, S. Mura, D. Brambilla, N. Mackiewicz and P. Couvreur, Chem. Soc. Rev., 2013, 42, 1147-1235.

32 D. Horák, N. Semenyuk and F. Lednický, J. Polym. Sci., Part A-1: Polym. Chem., 2003, 41, 1848-1863.

33 Y. Qiu, R. Palankar, M. Echeverría, N. Medvede, S. E. Moya and M. Delcea, Nanoscale, 2013, 5, 12624-12632.

34 J. Zeng, B. Jia, R. Qiao, C. Wang, L. Jing, F. Wang and M. Gao, Chem. Commun., 2014, 50, 2170-2172.

35 B. Li, J. Yang, L. Ma, F. Li, Z. Tu and C. Gao, Tissue Eng., Part A, 2014, 20, 1-11.

36 W. Wang, B. Li, J. Yang, L. Xin, Y. Li, H. Yin, Y. Qi, Y. Jiang, H. Ouyang and C. Gao, Biomaterials, 2010, 31, 8964-8973.

37 P. G. Waser, U. Muller, J. Kreuter, S. Berger, K. Munz, E. Kaiser and B. Pfluger, Int. J. Pharm., 1987, 39, 213-227.

38 M. Nishikawa, F. Staud, S. Takemura, Y. Takakura and M. Hashida, Biol. Pharm. Bull., 1999, 22, 214-218.

39 M. Chanana, P. Rivera Gil, M. A. Correa-Duarte, L. M. Liz-Marzán and W. J. Parak, Angew. Chem., Int. Ed., 2013, 52, 4179-4183. 